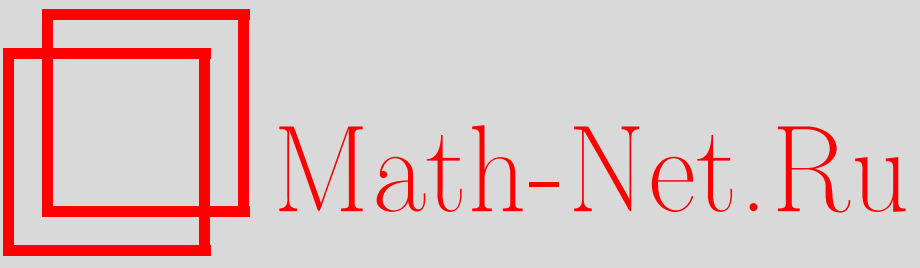

Б. Г. Авербух, Формализация понятия “система счисления", УМН, 1999, том 54, выпуск 1, 253-254

DOI: https://doi.org/10.4213/rm124

Использование Общероссийского математического портала Math-Net.Ru подразумевает, что вы прочитали и согласны с пользовательским соглашением

http://www.mathnet.ru/rus/agreement

Параметры загрузки:

IP : 3.89 .185 .249

26 апреля 2023 г., 14:49:33

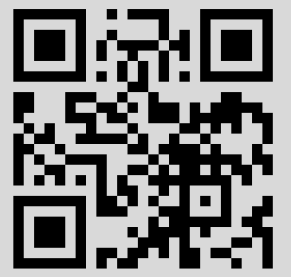




\title{
ФОРМАЛИЗАЦИЯ ПОНЯТИЯ “СИСТЕМА СЧИСЛЕНИЯ”
}

\author{
Б. Г. Аверьух
}

Понятие системы счисления, известное ныне в частных случаях, определяется ниже для тополого-алгебраической системы с одной ассоциативной операцией.

1. Пусть $(\mathscr{G}, \cdot)$ - хаусдорфова топологическая $n$-полугруппа, $\mathscr{B}$ - ее подмножество, называемое ниже вычислительным базисом, и $\langle\mathscr{B}\rangle$ - его линейная оболочка. Обозначим через $\widehat{\mathscr{D}}_{0}$ множество конечных или бесконечных сходящихся в $\mathscr{G}$ произведений элементов из $\mathscr{B}$. Если такое произведение конечно, то число его сомножителей имеет вид $s_{k}=1+k(n-1)$, где $k \in \mathbb{N}_{0}$. Каждому произведению $b_{1} b_{2} \cdots$ сопоставим последовательность из элементов $a_{k}=b_{1} \cdots b_{s_{k}}$, называемую его последовательностью приближений; для конечного произведения такая последовательность считается бесконечной, но стационарной. Пусть, далее, $(\widehat{\mathscr{D}}$, о) есть свободная $n$-полугруппа, построенная на множестве $\widehat{\mathscr{D}}_{0}$. Каждый элемент из $\widehat{\mathscr{D}}$, называемый ниже разложением, это упорядоченный набор из $s_{k}, k \in \mathbb{N}_{0}$, произведений указанного выше вида, называемых его компонентами, и ему соответствует упорядоченный набор их последовательностей приближений. Разложение называется конечным, если каждая его компонента является конечным произведением; всюду плотная под- $n$-полугруппа конечных разложений обозначается ниже через $\widehat{\mathscr{D}}_{f}$.

Каждому $s_{k}$-компонентному разложению $B$ с последовательностями приближений компонент $\left\{a_{i r}\right\}$ и каждому набору $\left\{r_{i}\right\}$, где $i \in \overline{1, s_{k}}$, неотрицательных целых чисел соответствует произведение $\prod_{i} a_{i r_{i}}$. Получается сходящаяся в $\mathscr{G}$ направленность элементов из $\langle\mathscr{B}\rangle$, назьваемая направленностью приближений разложения $B$. Она индексирована направленным множеством $\mathbb{N}_{0}^{s_{k}}$, где порядок задается условием: $\left(r_{1}^{\prime}, r_{2}^{\prime}, \ldots\right) \leqslant\left(r_{1}^{\prime \prime}, r_{2}^{\prime \prime}, \ldots\right)$, если $r_{i}^{\prime} \leqslant r_{i}^{\prime \prime}$ для всех $i$. Сопоставляя каждому разложению предел его направленности приближений, получаем гомоморфизм $n$-полугрупп $\widehat{\pi}:(\widehat{\mathscr{D}}, \circ) \rightarrow(\mathscr{G}, \cdot) ;$ при этом $\widehat{\pi}\left(\widehat{\mathscr{D}}_{f}\right)=\langle\mathscr{B}\rangle$. Образ $\widehat{\pi}(\widehat{\mathscr{D}})$ обозначим через $\mathscr{X}$.

2. На множестве $\widehat{\mathscr{D}}$ вводится топология. Сначала каждому конечному набору $V=\left\{\mathscr{V}_{1}, \ldots\right.$, $\left.\mathscr{V}_{r}\right\}$ открытых подмножеств из $\mathscr{G}$ сопоставляется подмножество $\widehat{V} \subset \widehat{\mathscr{D}}_{0}$. Однокомпонентное разложение $B$ с последовательностью приближений $\left\{a_{k}\right\}$ и предельным элементом $g$ принадлежит $\widehat{V}$, если $a_{k} \in \mathscr{V}_{k}$ при $k<r, a_{k}, g \in \mathscr{V}_{r}$ при $k \geqslant r$. Семейство $\{\widehat{V}\}$ образует базу топологии в $\widehat{\mathscr{D}}_{0}$. Она распространяется на множество $\widehat{\mathscr{D}}_{k} s_{k}$-компонентных разложений как на произведение $\left(\widehat{\mathscr{D}}_{0}\right)^{s_{k}}$, а на $\widehat{\mathscr{D}}$ как на сумму $\bigoplus_{r} \widehat{\mathscr{D}}_{r}$. Если топология в $\mathscr{G}$ равномерна, то равномерна и эта топология в $\widehat{\mathscr{D}}$. Операция о в $\widehat{\mathscr{D}}$ непрерьвна, а если в $\mathscr{G}$ задана равномерная структура, то и равномерно непрерывна в соответствующей структуре на $\widehat{\mathscr{D}}$. Гомоморфизм $\widehat{\pi}$ непрерывен, а если $(\mathscr{G}, \cdot)$ - равномерно непрерывная $n$-полугруппа, то он равномерно непрерывен.

3. Следующий шаг состоит во введении эквивалентности $\sim_{a}$, названной алгебраической. В классических случаях она соответствует той неоднозначности, с которой конечные отрезки записи чисел преобразуются в разложения по вычислительному базису. Она определяется как транзитивное замькание отношения, состоящего из пар разложений, чьи направленности приближений имеют общую конфинальную подпоследовательность. Отношение $\sim a$ является конгруенцией в $(\widehat{\mathscr{D}}, \circ)$, и мы обозначим через $(\mathscr{D}, *)$ фактор- $n$-полугруппу $(\widehat{\mathscr{D}}, \circ) / \sim_{a}$.

Теорема 1. Если операция · в $\mathscr{G}$ коммутативна, то коммутативна и операция * в $\mathscr{D}$.

4. Тонким моментом является введение топологии в $\mathscr{D}$. Она зависит от того, в качестве объекта какой категории рассматривается $\mathscr{G}$. Основными (но не единственными и не взаимоисключающими) являются, вероятно, следующие три случая: случай U), охватывающий абелевы топологические группы, когда $n$-полугруппа $(\mathscr{G}, \cdot)$ равномерно непрерывна; затем включающий неабелевы группы случай $\mathrm{CR}$ ), когда она только непрерывна, но топология на $\mathscr{G}$ равномерна, и 
общий случай, обозначаемый через Т). В случае U) топология вводится как факторная в категории UNIF, в случае CR) - как факторная в категории COMPL.REG. и в случае T) - как факторная в категории ТОР. Алгебраически эквивалентные разложения имеют равные предельные элементы, а для конечных разложений это условие также и достаточно для их алгебраической эквивалентности. Значит, определено отображение $\pi: \mathscr{D} \rightarrow X$, индуцированное отображением $\widehat{\pi}$.

5. Пространства $\widehat{\mathscr{D}}$ и $\mathscr{D}$, вообще говоря, не отделимы, поэтому вводится еще одно отношение эквивалентности $\sim_{t}$, названное топологическим. Оно отождествляет элементы из $\mathscr{D}$, каждый из которых лежит в любой окрестности другого. Это конгруенция, и топология в фактор- $n$-полугруппе $\left(\mathscr{D}^{\prime}, \times\right)$ вводится как факторная в соответствующей категории. Отображение $\pi$ индуцирует гомоморфизм $\pi^{\prime}:\left(\mathscr{D}^{\prime}, \times\right) \rightarrow(\mathscr{X}, \cdot)$. В случае U) операции в $\mathscr{D}$ и в $\mathscr{D}^{\prime}$ равномерно непрерывны, в двух других случаях они непрерывны по каждому аргументу. Отображения $\pi$ и $\pi^{\prime}$ являются морфизмами категории, соответствующей рассматриваемому случаю, причем их ограничения на подпространствах классов конечных разложений биективны.

Ключевым является следующий пример. Пусть $(\mathscr{G}, \cdot)=\left(R_{+},+\right)$и $\mathscr{B}=\left\{b_{k}\right\}$, где $b_{0}=1$ и $b_{i} / b_{i+1} \in \mathbb{N}$; этот случай рассмотрен в четвертой главе "Общей топологии" Н. Бурбаки. Тогда каждое разложение любого числа алгебраически эквивалентно одному из названных "употребительными" в русском переводе трактата. Классы "употребительных" разложений одного и того же элемента топологически эквивалентны (например, десятичные дроби с девяткой в периоде эквивалентны соответствующим конечным дробям), а отображение $\pi^{\prime}$ является изоморфизмом равномерно непрерывных полугрупп.

6. ОПРЕДЕЛЕНИЕ. $\mathscr{B}$-системой счисления в $(\mathscr{G}, \cdot)$ называется всякое сечение отображения $\pi$, определенное на $\mathscr{X}$ и сопоставляющее каждому элементу из $\langle\mathscr{B}\rangle$ класс его конечных разложений.

Естественным требованием к системе счисления является ее непрерьвность в случаях CR) и Т) и равномерная непрерывность в случае $\mathrm{U})$.

Теорема 2. Пусть $(\mathscr{G}, \cdot)$ - абелева топологическая группа (случай U)), а вычислительный базис $\mathscr{B}$ замкнут относительно перехода $к$ обратным әлементам. Тогда всякая В̈В-система счисления либо равномерно непрерывна, либо разрывна на каждом әлементе из $\langle\mathscr{B}\rangle$.

Пусть теперь $C$ - вполне регулярное пространство в случае $\mathrm{CR}$ ) или равномерное в случае $\mathrm{U}$ ), а $\lambda:\langle\mathscr{B}\rangle \rightarrow C$ - некоторое отображение. Мы назовем его продолжаемым до морфизма на $\widehat{\mathscr{D}}$, если существует такой морфизм $\mu: \widehat{\mathscr{D}} \rightarrow C$, что $\left.\mu\right|_{\widehat{\mathscr{D}}_{f}}=\left.\lambda \circ \widehat{\pi}\right|_{\widehat{\mathscr{D}}_{f}}$.

Tеорема 3. В случаях CR) и U) следующие утверждения әквивалентны:

i) непрерывная в случае $\mathrm{CR}$ ) или равномерно непрерывная в случае U) $\mathscr{B}$-система счисления существует;

ii) отображение $\pi^{\prime}$ является изоморфизмом топологических (соответственно, равномерно непрерывных) n-полугрупп;

iii) для любого C такого, как выше, всякое продолжаемое до морфизма на $\widehat{\mathscr{D}}$ отображсение само является морфизмом и допускает непрерывное продолжение на $\mathscr{X}$.

Автор благодарит А. В. Михалева за внимание к этой работе. 